\title{
Using the Selective Functional Movement Assessment for the Evaluation of Dancers' Functional Limitations and Dysfunctions: A Critically Appraised Topic
}

\author{
Victoria Fauntroy, Marcie Fyock, Jena Hansen-Honeycutt, Esther Nolton, \\ and Jatin P. Ambegaonkar
}

\begin{abstract}
Clinical Scenario: Dancers participate in a functionally demanding activity. Athletic participation typically requires the completion of a preparticipation examination, which involves a functional movement screen offering insight into potential injury recognition. The Selective Functional Movement Assessment (SFMA) was created to measure the status of movement-patternrelated pain and dysfunction using regionally interdependent movement to aggravate symptoms and exhibit limitations and dysfunctions. Still, a functional assessment has not been identified to recognize potential dysfunctions or limitations in this population. Clinical Question: Does the use of the SFMA improve overall evaluation of dancers by providing more information on a dancer's overall functional ability and limitations? Summary of Key Findings: The literature search discovered 12 studies and 3 books in which 4 studies were included ( 2 case reviews, 1 case report, and 1 original research study) based on the inclusion and exclusion criteria. Three of the studies provided clinical case studies utilizing the SFMA to improve the patient's dysfunctions, whereas 1 study examined the intrarater and interrater reliability of the SFMA. In 3 studies, participants displayed less movement dysfunction. The authors from 3 of the studies agreed the SFMA was a valuable tool for clinicians to use during evaluations, as it provided a more holistic view of the patient, discovering dysfunctional movement patterns that may better identify the source of injury. Clinical Bottom Line: Low-quality evidence, defined as poorly designed case studies, case series, and cohort studies, exist that supports improvement of overall evaluations when utilizing the SFMA. Although the studies were considered low-quality evidence, each included study displayed an effective use of the SFMA as an overall evaluation that correctly identified dysfunctional movement patterns. Strength of Recommendation: Grade C evidence exists that the SFMA contributes to the functional evaluation used in dancers.
\end{abstract}

Keywords: SFMA, athlete, regional interdependence, preparticipation examination

\section{Clinical Scenario}

Historically, athletic participation required the completion of a functional movement screening during the preparticipation examination, which provided valuable information to clinicians regarding injury prevention. However, in recent years, functional movement screenings have begun to be incorporated in the preparticipation examination, providing additional injury recognition information. Recently, the Selective Functional Movement Assessment (SFMA) was developed by a team of clinicians and has its origins in the functional movement screen (FMS) to measure the status of movement-pattern-related pain and dysfunction. ${ }^{1,2}$ The SFMA uses regionally interdependent movement to exacerbate symptoms leading to the manifestation of limitations and dysfunctions, with the resulting information relating to the patient's chief complaint in the attempt to create more functional movement and decrease injury. 2,3

Traditionally, clinicians have utilized a biomedical model of disease as the basis of evaluation, focusing solely on a biological reason to explain injury or illness. ${ }^{4}$ The biomedical model often focuses treatment at the location of pain or chief complaint and

Fauntroy, Fyock, Nolton, and Ambegaonkar are with the Sports Medicine Assessment Research and Testing (SMART) Laboratory, George Mason University, Manassas, VA, USA. Hansen-Honeycutt is with the Department of Dance, George Mason University, Fairfax, VA, USA. Ambegaonkar (jambegao@gmu.edu) is corresponding author. rarely considers other anatomical locations as possible reasons for the injury. The limited information provided by this model narrowed the available treatment options. Recently, a paradigm shift to expand on the biomedical model and include other factors or regions that may contribute to the individuals' complaint has occurred. ${ }^{4}$

The regional interdependence (RI) model describes the concept that a patient's primary musculoskeletal symptom(s) may be directly or indirectly related or influenced by impairments from various body regions and systems regardless of proximity to the primary symptom(s). ${ }^{4}$ The RI model presents an opportunity for clinicians to identify remote dysfunctions that may be affecting or causing the patient's chief complaint, which is an integral role in the SFMA. The SFMA is a clinical assessment that is designed to systematically identify causes of movement dysfunction while taking pain into consideration using an algorithmic approach. ${ }^{1}$ Guided by the RI model, the SFMA can be used by the clinician to identify dysfunctions in different parts of the body and determine their relationship in the overall injury.

The SFMA has been used to identify remote dysfunctions through the utilization of 7 top-tier (ie, basic movement) assessments: cervical patterns-flexion, extension, and rotation (right $[\mathrm{R}]+$ left $[\mathrm{L}]$ ); upper-extremity patterns: medial rotation-extension pattern $(\mathrm{R}+\mathrm{L})$ and lateral rotation-abduction pattern $(\mathrm{R}+\mathrm{L})$; multisegmental patterns: multisegmental flexion; multisegmental extension; multisegmental rotation $(\mathrm{R}+\mathrm{L})$; single-leg stance $(\mathrm{R}+\mathrm{L})$; and overhead squat. ${ }^{5}$ The results of the SFMA are separated into 4 categories: functional nonpainful, functional painful, 
dysfunctional nonpainful, and dysfunctional painful. ${ }^{2}$ The SFMA has also been used to evaluate athletes in various sports such as soccer, weight lifting, running, and baseball. ${ }^{5-7}$

However, a functional assessment has not been identified for dancers. Dancers participate in a physically demanding activity requiring extreme functional movement. As with any sport, dancers suffer injuries resulting from the demands required by their activity and will often continue to train while in pain. Specifically, dancers have high rates of injury, with $80 \%$ to $97 \%$ of university, professional ballet, and modern dancers reporting at least 1 musculoskeletal injury per year. ${ }^{8}$ Most of these injuries occur in the lower-extremity or lumbar spine because of overuse or repetitive motions. ${ }^{8-10}$ Thus, there is a necessity for the critical appraisal of research to examine the effectiveness of using the SFMA in the evaluation process to assess dancers' functional movement to decrease injury incidence. If the true dysfunction causing the injury is not addressed, other regions of the body will compensate to produce the movement required for the activity. ${ }^{5}$ Compensation may only lead to further injuries and pain for the dancer.

\section{Focused Clinical Question}

Does the use of the SFMA improve overall evaluation of dancers by providing more information on the dancers' overall functional ability and limitations?

\section{Summary of Search, "Best Evidence" Appraised, and Key Findings}

- Twelve studies and 3 books were identified during the electronic search; 2 case reviews, 1 case report, and 1 original research study fulfilled the inclusion and exclusion criteria and were included in this critically appraised topic (CAT) (Table 1).

- Three of the included studies provided clinical case studies using the SFMA with appropriate treatment interventions to improve the patient's dysfunctions. ${ }^{5,6,10}$

- One included study examined the intrarater and interrater reliability of the SFMA using both the categorical scoring tool and criterion checklist in raters with various levels of experience. $^{11}$

- In 3 of the studies included in this CAT, all dysfunctional movement demonstrated by the participants was resolved and completed in a more functional manner. ${ }^{5,6,10}$

- In the last included study, the authors found that the intrarater and interrater reliability of categorical scoring and criterion checklist scoring of the SFMA were reflective of level of experience. Specifically, in a healthy population, the SFMA was most reliable between sessions when performed by a single-experienced rater. ${ }^{11}$

- The authors from the included studies that used the SFMA agreed that the SFMA was a beneficial tool for clinicians during evaluations because it provided a more integrated view of the patient, discovering dysfunctions that may have been the cause of injury. $5,6,10,11$

\section{Clinical Bottom Line}

There is low-quality evidence for the improvement of overall evaluations using the SFMA based on the level 4 evidence of

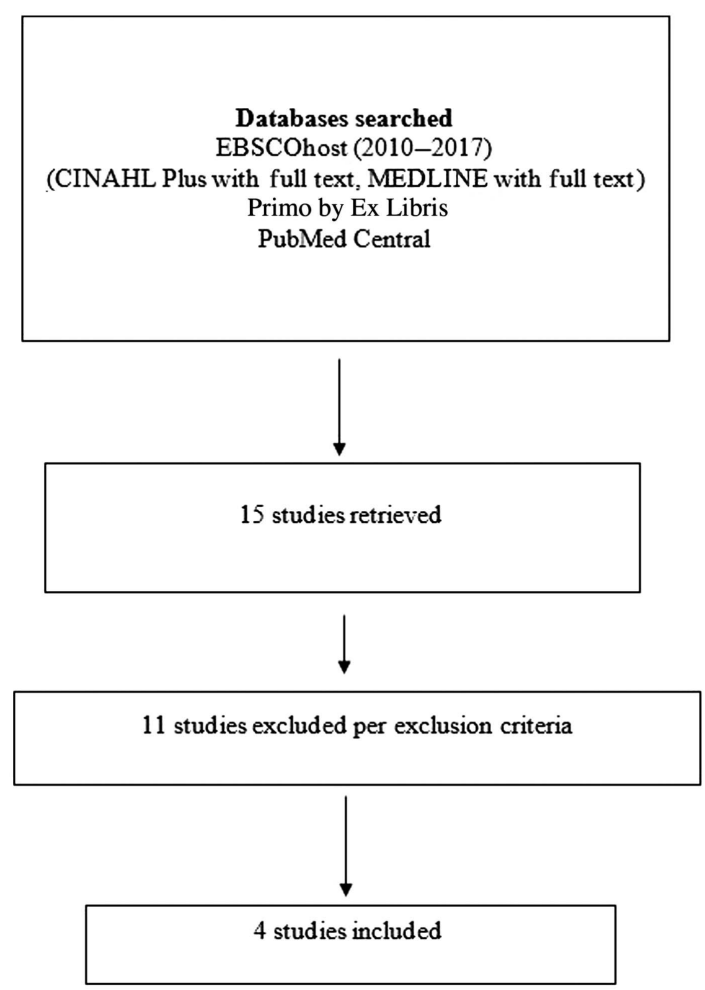

Figure 1 - Summary of search strategy.

the studies reviewed for this CAT. Although the studies were considered low-quality evidence, each study concluded that the SFMA correctly identified dysfunctions and limitations causing the injury, which assisted the clinicians in the creation of proper rehabilitation programs for their patients. Nevertheless, because of limited original research and/or high-quality studies that discuss the SFMA only, this CAT reviewed studies deemed poor-quality evidence to answer the clinical question.

\section{Strength of Recommendation}

Grade C evidence exists that the SFMA improves the overall evaluation of dancers by contributing additional information on the comprehensive functional ability and limitations of the dancer. The Oxford Centre for Evidence-Based Medicine defines level 4 evidence as poorly designed case studies, case series, and cohort studies. A grade of $\mathrm{C}$ is suggested by the Oxford Centre for Evidence-Based Medicine for level 4 studies. ${ }^{12}$

\section{Search Strategy}

An electronic search was conducted in September and October 2017 (Figure 1).

\section{Terms Used to Guide Search Strategy}

- Patient/Client Group: All dancers (ie, no specific discipline)

- Intervention (or Assessment): Selective Functional Movement Ässessment (SFMA) 
- Comparison: Athletes participating in competitive sport (ie, team sports)

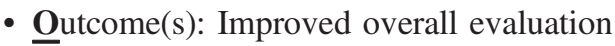

\section{Sources of Evidence Searched (Databases)}

- CINAHL Plus

- MEDLINE

- PubMed Central

- Primo by Ex Libris

- Supplementary resources retrieved via reference list review and web searches

\section{Inclusion and Exclusion Criteria}

\section{Inclusion Criteria}

- Restricted to studies describing SFMA and its uses in dancers

- Restricted to studies in the English language

- Restricted to studies published within in the last 7 years (2010-2017)

\section{Exclusion Criteria}

- Systematic reviews, meta-analyses, clinical reviews, books, CATs, and clinical commentaries

\section{Results of Search}

Four pertinent studies were discovered and classified as presented in Table 1 . The validity of the selected studies was assessed using the Physiotherapy Evidence Database (PEDro) scale to score each study based on certain criteria. The included studies also received a letter grade and level of evidence number as defined by the Oxford Centre for Evidence-Based Medicine. ${ }^{12}$

\section{Best Evidence}

The ensuing studies were recognized as the "best" evidence and chosen for inclusion in the CAT. The reasons for selecting these studies were as follows:

- Met all inclusion and exclusion criteria

- Discussed SFMA as an evaluation tool

Table 1 Summary of Study Designs of Articles Using the Selective Functional Movement Assessment to Measure the Status of Movement Pattern-Related Pain and Dysfunction

\begin{tabular}{llcl}
\hline Level of & $\begin{array}{l}\text { Study design/ } \\
\text { methodology } \\
\text { of articles } \\
\text { retrieved }\end{array}$ & $\begin{array}{l}\text { Number } \\
\text { located }\end{array}$ & Author (year) \\
\hline 4 & Case report & 1 & $\begin{array}{l}\text { Goshtigian and } \\
\text { Swanson }(2016)^{5} \\
\text { Krzyzanowicz et al } \\
(2015)^{10} \\
\text { Mokha et al }(2015)^{6} \\
\text { Glaws et al }(2014)^{11}\end{array}$ \\
\hline $\mathrm{b}$ & Case review & 2 & \\
\hline
\end{tabular}

\section{Summary of Best Evidence}

Table 2 describes characteristics of included studies using the SFMA to measure the status of movement pattern-related pain and dysfunction.

\section{Implications for Practice, Education, and Future Research}

Dance is a physically demanding, athletic activity requiring elevated functional ability to repeatedly perform at a prominent level. For example, professional dancers routinely participate in technique classes during most of any given day, followed by rehearsals and performances through the late afternoons and evenings. Preparticipation exams are frequently performed in athletic sport participation, yet this is not commonly performed for dancers. A functional screening for dancers may be of benefit to observe the movement patterns of the dancer. ${ }^{10}$ Krzyzanowicz et $\mathrm{al}^{10}$ noted that the SFMA correctly identified dysfunctions leading to sacroiliac joint pain in 3 dancers. The authors suggested the use of the SFMA to other clinicians as part of their evaluation process and to help with the proper creation of rehabilitation programs to correct the dysfunction.

The SFMA incorporates the "breakout," which is utilizing a movement pattern isolation map to establish probable causes for the dysfunction when a movement is not categorized as functional nonpainful. ${ }^{10}$ The dysfunction is divided into 3 categories: (1) tissue extensibility dysfunction (TED), (2) joint mobility dysfunction (JMD), or (3) stability or motor control dysfunction (SMCD). ${ }^{10}$ A tissue extensibility dysfunction recognizes tissues that cross more than 1 joint or are multiarticular (ie, neural tension, fascial tension, or muscle shortening). ${ }^{2,10} \mathrm{~A}$ joint mobility dysfunction classifies the articular surfaces and the contractile and noncontractile connecting spinal articular segments that exhibit decreased mobility (ie, osteoarthritis, adhesive capsulitis, fusion). ${ }^{2}$ A stability or motor control dysfunction is broken into either a stability dysfunction (ie, strength) or a motor control dysfunction (ie, neurological processing problem). ${ }^{2,10}$

Once the dysfunctions are recognized utilizing the SFMA breakout, therapeutic rehabilitation programs can be implemented that improve the dancer's dysfunctional nonpainful or dysfunctional painful movement to functional nonpainful patterns. Interestingly, Krzyzanowicz et al ${ }^{10}$ noticed that each dancer exhibited dysfunction in the multisegmental flexion, single-leg stance, and overhead squat patterns. Goshtigian and Swanson ${ }^{5}$ discovered their college soccer player displayed dysfunction in the cervical rotation $(\mathrm{R}+\mathrm{L})$, medial rotation-extension, overhead squat, and all multisegmental (flexion, extension, rotation $[\mathrm{R}+\mathrm{L}]$ ) patterns. Similarly, Mokha et $\mathrm{al}^{6}$ recorded their college runner's demonstrated dysfunction in all multisegmental patterns (flexion, extension, rotation $[\mathrm{R}+\mathrm{L}])$. In these cases, the discovery of these dysfunctions contributed information that helped decrease the patient's chief complaint. The treatment administered to each patient was categorized based on the SFMA breakout findings. For example, patient 1 in the Krzyzanowicz et $\mathrm{al}^{10}$ study completed the rolling technique and other similar exercises to address her stability or motor control dysfunction, ${ }^{10}$ whereas the patient in the Goshtigian and Swanson ${ }^{5}$ study performed joint mobilizations to address his joint mobility dysfunction. The included case reviews and single case study provided vital information in this CAT that may allow for a more appropriate rehabilitation and treatment protocol. In the reviewed studies, all concluded the SFMA was beneficial and 


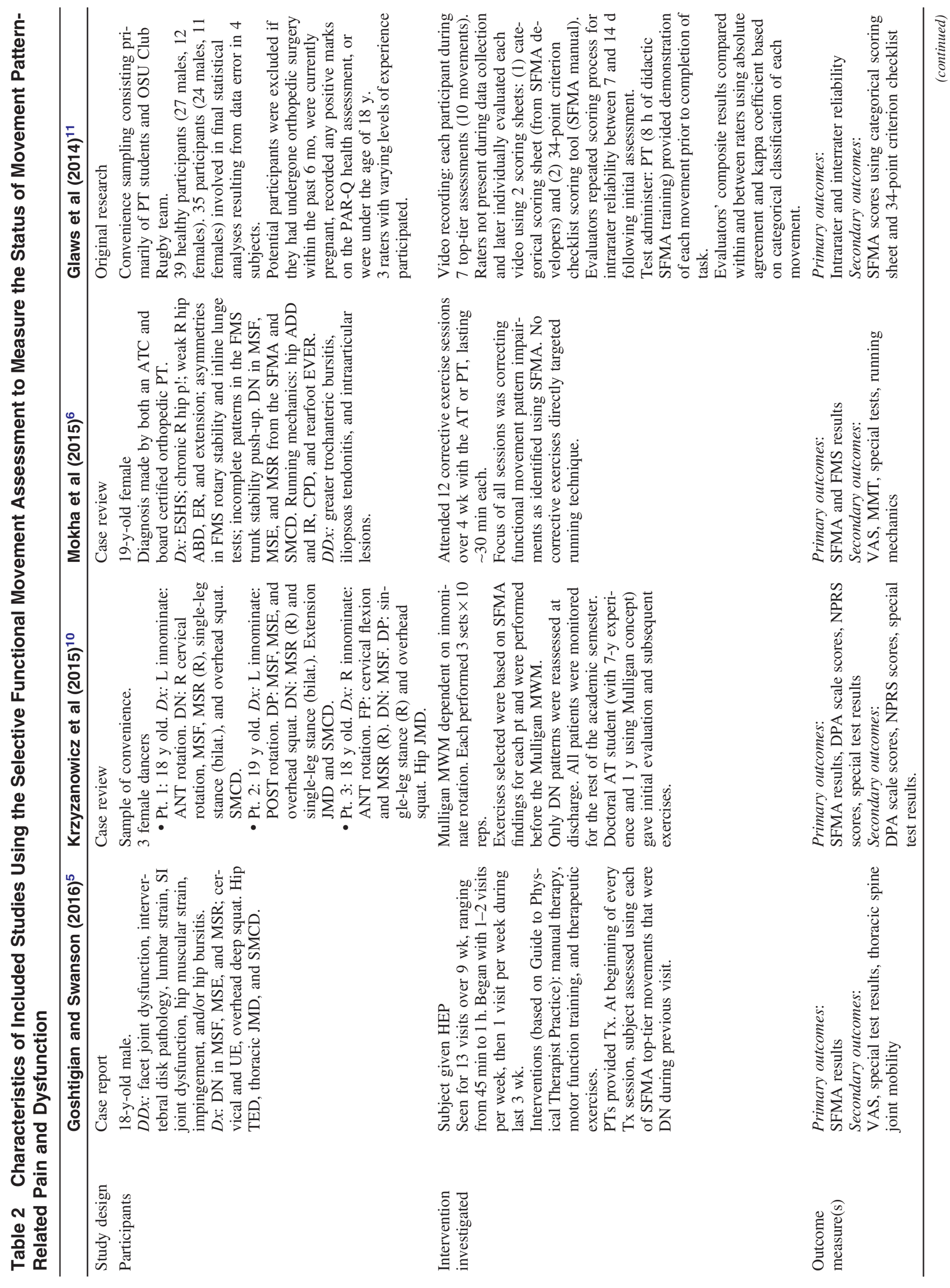




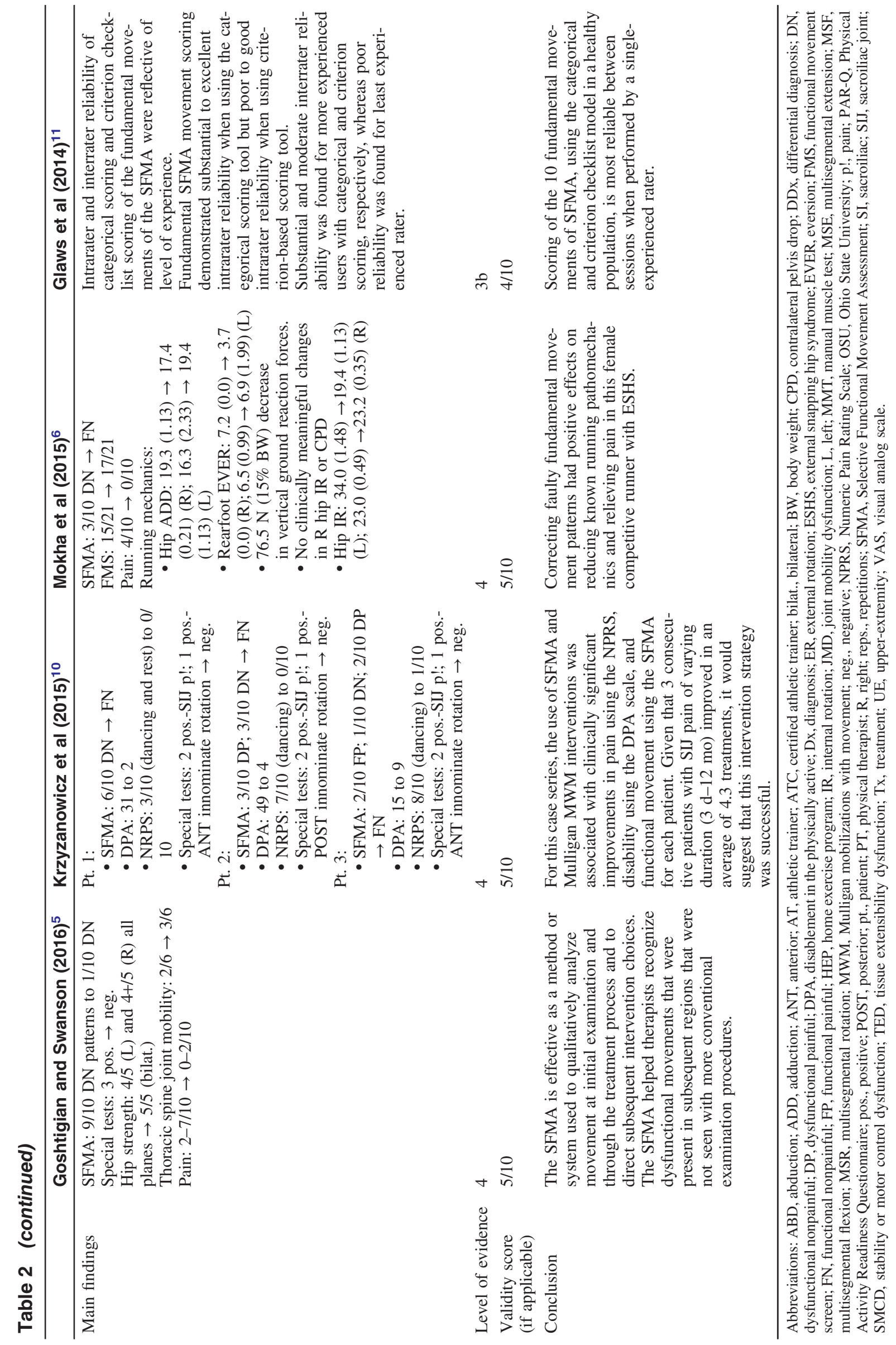


relevant to identifying both dance and other team sport athletes' movement dysfunction ${ }^{5,6,10,11}$ because the method incorporates the RI model to identify and treat dysfunctions in the body to provide holistic functional movement. ${ }^{4}$

Based on the conclusions of the authors in each of the included studies, there is moderate evidence to suggest that clinicians use the SFMA to improve a dancer's overall clinical evaluation. Although the studies were considered low-quality evidence, each included study displayed effective use of the SFMA as an overall evaluation that correctly identified dysfunctional movement patterns. Additional research needs to be conducted on the efficacy of the SFMA and its associated treatment outcomes, as well as the validity of the SFMA itself. Future studies should specifically concentrate on the use of the SFMA in the dance populations, as there is little published information about a dance-specific functional screening. Finally, this CAT should be reviewed in 2 years to determine whether additional best research evidence has been published that could aid in answering the focused clinical question about examining dancers' functional capacities with the goals of decreasing their injury risk and improving their performance.

\section{Acknowledgments}

The authors declare no conflict of interest.

\section{References}

1. Cook G, Burton L, Hoogenboom BJ, Voight M. Functional movement screening: the use of fundamental movements as an assessment of function-part 1. Int J Sports Phys Ther. 2014;9(3):396-409. PubMed ID: 24944860

2. Cook G. Movement: Functional Movement Systems: Screening, Assessment and Corrective Strategies. Aptos, CA: Target Publications; 2010.

3. Rhinehart AJ, Schroeder KM, May J, Baker R, Nasypany A, Ambegaonkar JP. Movement assessment: techniques and possible integration into clinical practice. Int J Athl Ther Train. 2015;20(6): 5-9. doi:10.1123/ijatt.2015-0021
4. Sueki DG, Cleland JA, Wainner RS. A regional interdependence model of musculoskeletal dysfunction: research, mechanisms, and clinical implications. J Man Manip Ther. 2013;21(2):90-102. PubMed ID: 24421619 doi:10.1179/2042618612Y.0000000027

5. Goshtigian GR, Swanson BT. Using the selective functional movement assessment and regional interdependence theory to guide treatment of an athlete with back pain: a case report. Int J Sports Phys Ther. 2016;11(4):575-595. PubMed ID: 27525182

6. Mokha GM, Sprague PA, Rodriguez R, Gatens DR. Functional movement pattern training improves mechanics in a female runner with external snapping hip syndrome. Int J Athl Ther Train. 2015; 20(1):25-33. doi:10.1123/ijatt.2014-0095

7. Busch AM, Clifton DR, Onate JA, Ramsey VK, Cromartie F. Relationship of preseason movement screens with overuse symptoms in collegiate baseball players. Int J Sports Phys Ther. 2017;12(6): 960-966. PubMed ID: 29158957 doi:10.26603/ijspt20170960

8. Davenport KL, Air M, Grierson MJ, Krabak BJ. Examination of static and dynamic core strength and rates of reported dance related injury in collegiate dancers: a cross-sectional study. J Dance Med Sci. 2016; 20(4):151-161. doi:10.12678/1089-313X.20.4.151

9. Allen N, Nevill AM, Brooks JHM, Koutedakis Y, Wyon MA. The effect of a comprehensive injury audit program on injury incidence in ballet: a 3-year prospective study. Clin J Sport Med. 2013;23(5): 373-378. PubMed ID: 23558333 doi:10.1097/JSM.0b013e31828 $87 \mathrm{f} 32$

10. Krzyzanowicz R, Baker R, Nasypany A, Gargano F, Seegmiller J. Patient outcomes utilizing the selective functional movement assessment and mulligan mobilizations with movement on recreational dancers with sacroiliac joint pain: a case series. Int J Athl Ther Train. 2015;20(3):31-37. doi:10.1123/ijatt.2014-0066

11. Glaws KR, Juneau CM, Becker LC, Di Stasi SL, Hewett TE. Intraand inter-rater reliability of the selective functional movement assessment (SFMA). Int J Sports Phys Ther. 2014;9(2):195-207. PubMed ID: 24790781

12. Oxford Centre of Evidence-Based Medicine. CEBM levels of evidence 2.1. 2011. http://www.cebm.net/wp-content/uploads/2014/ 06/CEBM-Levels-of-Evidence-2.1.pdf. Accessed December 14, 2017. 